\title{
ORTHANT SPANNING SIMPLEXES WITH MINIMAL VOLUME
}

\author{
MICHELE ELIA
}

\author{
Received 23 October 2002
}

\begin{abstract}
A geometry problem is to find an (n-1)-dimensional simplex in $\mathbb{R}^{n}$ of minimal volume with vertices on the positive coordinate axes, and constrained to pass through a given point $A$ in the first orthant. In this paper, it is shown that the optimal simplex is identified by the only positive root of a $\left(2^{n}-1\right)$-degree polynomial $p_{n}(t)$. The roots of $p_{n}(t)$ cannot be expressed using radicals when the coordinates of $A$ are transcendental over $\mathbb{Q}$, for $3 \leq n \leq 15$, and supposedly for every $n$. Furthermore, limited to dimension 3 , parametric representations are given to points $A$ to which correspond triangles of minimal area with integer vertex coordinates and area.
\end{abstract}

2000 Mathematics Subject Classification: 26B15, $11 D 99$.

1. Introduction. A geometry problem is to find an (n-1)-dimensional simplex $\mathbf{S}$ in $\mathbb{R}^{n}$ of minimal volume, whose $n$ vertices are points $V_{i}=\left(0, \ldots, 0, v_{i}\right.$, $0, \ldots, 0)$, with $v_{i}>0$ on the $i$ th coordinate axis and $1 \leq i \leq n$, which is constrained to pass through a given point $A=\left(a_{1}, a_{2}, \ldots, a_{n}\right) \in \mathbb{R}^{n}$ in the positive orthant $a_{i}>0,1 \leq i \leq n$. Throughout the paper, this problem, which was proposed and numerically solved in [8], will be referred to as the optimal simplex problem. The simplex belongs by definition to a plane with "segmental" equation $\sum_{i=1}^{n} x_{i} / v_{i}=1$, that is,

$$
\sum_{i=1}^{n} \frac{a_{i}}{v_{i}}=1
$$

and its volume (see [7, pages 123-124]) is

$$
S=\frac{1}{(n-1) !} \sqrt{\prod_{i=1}^{n} v_{i}^{2} \sum_{j=1}^{n} \frac{1}{v_{j}^{2}}}
$$

The volume $S$ is the objective function of a constrained optimization problem with constraint (1.1) and $v_{i}>0, i=1, \ldots, n$. A standard way to look for a solution with objective function of the form (1.2) is to apply the Lagrange multiplier method to the logarithm of this function, namely,

$$
L=\ln S+\lambda \sum_{i=1}^{n} \frac{a_{i}}{v_{i}}=-\ln (n-1) !+\frac{1}{2} \ln \sum_{j=1}^{n} \frac{1}{v_{j}^{2}}+\sum_{i=1}^{n} \ln v_{i}+\lambda \sum_{i=1}^{n} \frac{a_{i}}{v_{i}},
$$


where $\lambda$ is computed to satisfy the constraint (1.1). The derivatives of $L$ with respect to each $v_{i}$

$$
\frac{\partial L}{\partial v_{i}}=\left(\frac{1}{v_{i}}-\frac{1}{\sum_{j=1}^{n}\left(1 / v_{j}^{2}\right)} \frac{1}{v_{i}^{3}}\right)-\lambda \frac{a_{i}}{v_{i}^{2}}, \quad i=1, \ldots, n,
$$

equated to 0 give $n$ conditions, which are sufficient to specify the $n$ unknowns $v_{i}$. The value $\lambda=n-1$ of the Lagrange multiplier is obtained from the sum $\sum_{j=1}^{n} v_{i}\left(\partial L / \partial v_{i}\right)=0$. Thus, setting $1 / t=\sum_{j=1}^{n}\left(1 / v_{j}^{2}\right) \geq 0, n$ quadratic polynomials $Q_{i}(v)=v^{2}-(n-1) a_{i} v-t$ are obtained from the partial derivatives, each with a single positive root

$$
v_{i}=\frac{n-1}{2} a_{i}+\sqrt{t+\left(\frac{n-1}{2} a_{i}\right)^{2}}, \quad 1 \leq i \leq n,
$$

that identifies a vertex of the optimal simplex. The positive parameter $t$ turns out to be the only nonnegative zero of the function

$$
g(t)=\sum_{j=1}^{n} \frac{a_{j}}{v_{j}}-1=\sum_{j=1}^{n} \frac{a_{j}}{((n-1) / 2) a_{j}+\sqrt{t+\left(((n-1) / 2) a_{j}\right)^{2}}}-1 .
$$

Equations $g(t)=0,(1.5)$, and (1.2) offer a numerical way to compute vertices and volume of the optimal simplex $\mathbf{S}$ for any $A \in \mathbb{R}^{n}$ [8]. The only positive zero of $g(t)=0$ is a root of a polynomial $p_{n}(t)$ of degree $2^{n}-1$ whose coefficients are rational functions of $a_{i}$ 's obtained as follows. Let $\mathbb{G}\left(Q_{i}(v)\right)$ be the Galois group of $Q_{i}(v)$ which has order 2. Let $\sigma_{\mathfrak{s}}(g(t))$ denote the function

$$
\sigma_{\mathfrak{s}}(g(t))=\sum_{j=1}^{n} \frac{a_{j}}{\sigma_{j}\left(v_{j}\right)}-1
$$

associated to a specified sequence of Galois automorphisms $\mathfrak{s}=\left(\sigma_{1}, \sigma_{2}, \ldots, \sigma_{n}\right)$ and $\sigma_{i} \in \mathfrak{G}\left(Q_{i}(v)\right)$. Since the product $P(t)=\prod_{\mathfrak{s}} \sigma_{\mathfrak{s}}(g(t))$ over all $2^{n}$ sequences $\mathfrak{s}$ is a symmetric function of the roots of $Q_{i}(v)$, for every $i=1, \ldots, n$, then $P(t)=p_{n}(t) / t^{2^{n}-1}$ is a rational function over $\mathbb{Q}\left(a_{1}, \ldots, a_{n}\right)$. The coefficients of $p_{n}(t)$ belong to $\mathbb{Q}\left(a_{1}, \ldots, a_{n}\right)$.

Although the solutions of geometry problems are numerically computable, it is a common practice to look for closed form solutions expressed using radicals. Alongside the three classical Greek problems [9] and the construction of regular polygons [4], there are numerous other problems. For example, the problem of computing the distance between two circles in space [5] and the 
problem of computing the length of a tangential polygon [6]. The optimal simplex problem [8] is a problem of this sort. The simplest cases $n=2$ and 3 illustrate the situation for any $n>3$.

THE CASE $n=2$. The problem consists in finding the shortest line passing through $A=(a, b)$ with endpoints on the positive axes. A direct solution is well known [8]; however, using the method outlined above, the same cubic polynomial

$$
p_{2}(t)=t^{3}-3 b^{2} a^{2} t-\left(b^{2} a^{4}+b^{4} a^{2}\right),
$$

which has the positive root expressed by radicals

$$
t_{o}=b\left(a^{2} b\right)^{1 / 3}+a\left(a b^{2}\right)^{1 / 3}
$$

is obtained.

The length $\ell$ of the segment $V_{1} V_{2}$ is

$$
\ell=\left[\left(\frac{a}{2}+\sqrt{t+\frac{a^{2}}{4}}\right)^{2}+\left(\frac{b}{2}+\sqrt{t+\frac{b^{2}}{4}}\right)^{2}\right]^{1 / 2}=\sqrt{\left(a^{2 / 3}+b^{2 / 3}\right)^{3}} .
$$

THE CASE $n=3$. The problem consists in finding a triangle of smallest area, passing through $A=(a, b, c)$, with vertices on the positive axes. Using the same method, we obtain a 7-degree polynomial

$$
\begin{aligned}
p_{3}(t)= & t^{7}+4 \sigma_{1} t^{6}+6 \sigma_{1}^{2} t^{5}+4\left(\sigma_{1}^{3}-10 \sigma_{3}\right) t^{4} \\
& +\sigma_{1}\left(\sigma_{1}^{3}-128 \sigma_{3}\right) t^{3}-8 \sigma_{3}\left(17 \sigma_{1}^{2}+8 \sigma_{2}\right) t^{2} \\
& -16 \sigma_{3}\left(3 \sigma_{1}^{3}+8 \sigma_{1} \sigma_{2}-5 \sigma_{3}\right) t-64 \sigma_{3} \sigma_{1}\left(\sigma_{1} \sigma_{2}-\sigma_{3}\right),
\end{aligned}
$$

where $\sigma_{1}=a^{2}+b^{2}+c^{2}, \sigma_{2}=a^{2} b^{2}+b^{2} c^{2}+c^{2} a^{2}$, and $\sigma_{3}=a^{2} b^{2} c^{2}$ are elementary symmetric functions of $a^{2}, b^{2}$, and $c^{2}$.

In Section 2, it will be proved that $p_{3}(t)$ is not solvable by radicals when $a, b$, and $c$ are distinct and transcendental over $\mathbb{Q}$. The same unsolvability will also be proved for a large set of triples $a, b$, and $c$ of integers. As a partial counterpart, the converse Diophantine problem will be solved, namely, to find points $A$ with integer coordinates such that the corresponding optimal triangles have vertices with integer coordinates and integer areas.

2. Impossibility of solutions by radicals. In this section, two proofs of the unsolvability of $p_{3}(t)$ will be given. The first is better for proving the 
unsolvability of $p_{n}(t)$ for any $n>3$. The second is more appropriate for addressing the solvability question with points $A$ of integer coordinates.

We consider the polynomial $p_{3}(t)$ obtained by setting $a=1, b=4$, and $c=6$,

$$
\begin{aligned}
P_{3}(t)= & t^{7}+212 t^{6}+16854 t^{5}+572468 t^{4}+3982897 t^{3} \\
& -243196416 t^{2}-6543571968 t-63904628736 .
\end{aligned}
$$

This polynomial is easily checked to be irreducible over $\mathbb{Q}$. It is therefore unsolvable by radicals if its Galois group $\mathfrak{G}\left(P_{3}\right)$ is unsolvable. This group is obtained by applying a method described in [10, Volume I, page 190]. The basis of this technique is that the Galois group of an irreducible polynomial over $\mathbb{Q}$ includes the Galois groups of the same polynomial considered over finite Galois fields of prime order $p$. The Galois group of an irreducible polynomial over a Galois field is a cyclic group of order equal to its degree. Therefore, if an irreducible polynomial over $\mathbb{Q}$ splits into irreducible factors over a prime field $\mathrm{GF}(p)$, its Galois group will contain the cyclic Galois groups of these irreducible factors as subgroups.

THEOREM 2.1. The Galois group $\mathfrak{G}\left(P_{3}\right)$ is isomorphic to the symmetric group $S_{7}$, thus $P_{3}(t)$ is not solvable by radicals.

Proof. The irreducible polynomial $P_{3}(t)$ factors modulo 13 and 7 , respectively, as

$$
\begin{aligned}
& P_{3}(t)=\left(t^{6}+4 t^{5}+6 t^{4}+9 t^{2}+5 t+2\right) t \bmod 13 \\
& P_{3}(t)=\left(t^{2}+3 t+6\right)(t+1)^{3}(t+5)^{2} \bmod 7 .
\end{aligned}
$$

The presence of a 6-degree factor in the factoring modulo 13 implies that the Galois group of $P_{3}(t)$ over $\mathbb{Q}$ contains a cycle of 6 symbols, while the single 2-degree factor and 5 linear factors in the factoring modulo 7 imply that the Galois group contains a cycle of 2 symbols. The conclusion follows from a theorem [10, Volume I, page 191] stating that a transitive permutation group of $n$ objects containing a cycle of two symbols and an $(n-1)$-cycle is a symmetric group $S_{n}$, with $n=7$. Since $S_{7}$ is a nonsolvable group [10, volume I, page 149], then $P_{3}(t)$ cannot be solved by radicals.

A specialization principle incorporated in Galois theory itself proves that $p_{3}(t)$ is unsolvable by radicals over the coefficient field $\mathbb{Q}(a, b, c)$ when $a, b$, and $c$ are distinct and transcendental over $\mathbb{Q}$.

Proposition 2.2. Let $c_{1}, \ldots, c_{k}$ be transcendental over $\mathbb{Q}$. If a polynomial $p\left(x, c_{1}, \ldots, c_{k}\right)$ over $\mathbb{Q}$ is solvable by radicals with respect to $x$, then every polynomial obtained setting $c_{j_{1}}=\alpha_{1}, \ldots, c_{j_{h}}=\alpha_{h}$ with $\alpha_{1}, \ldots, \alpha_{h} \in \mathbb{Q}$, for some 
$h \leq k$, is solvable by radicals. On the contrary, if there is a set of rational numbers such that $p\left(x, \alpha_{1}, \ldots, \alpha_{k}\right)$ is not solvable by radicals, then $p\left(x, c_{1}, \ldots, c_{k}\right)$ is not solvable by radicals.

As a consequence of this proposition, a fortiori the roots of $p_{3}(t)$ cannot be computed by radicals when $a, b$, and $c$ are distinct and transcendental over $\mathbb{Q}$.

The fact observed with $P_{3}(t)$ is not extemporary. Any irreducible polynomial $p_{3}(t)$ obtained from a triple of integers $a, b$, and $c$ is actually unsolvable by radicals. To prove this, let a variable substitution $t=z^{2}-2 z a, b^{2}=x$, and $c^{2}=y$ be performed on $p_{3}(t)$. A 14-degree polynomial in $z$, which splits over the coefficient field into two polynomials of degree 7 , is obtained. Since each of these two factors is transformed into the other by a linear substitution $z \rightarrow-z+2 a$, for the following analysis it is indifferent which factor is retained:

$$
\begin{aligned}
q_{3}(z)= & z^{7}-4 a z^{6}+\left(2 y+6 a^{2}+2 x\right) z^{5}+\left(-4 a y-4 a^{3}-4 a x\right) z^{4} \\
& +\left(y^{2}+2 x y+a^{4}+x^{2}+2 a^{2} y+2 a^{2} x\right) z^{3}+8 a y x z^{2} \\
& -12 a^{2} y x z+8 a x y^{2}+8 a x^{2} y .
\end{aligned}
$$

Since $q_{3}(z)$ and $p_{3}(t)$ are related by the Tschirnhaus transformation $t=z^{2}-$ $2 z a$, both are solvable or unsolvable in the same way. The proof of Theorem 2.4 uses the following property, reported from [3] without proof.

Proposition 2.3 [3, page 266]. Let $f(z)$ be a polynomial of degree $n$ over $\mathbb{Q}$. The Galois group $\mathbb{6}(f)$ is isomorphic to a subgroup of the alternating group $A_{n}$ of degree $n$ if and only if the discriminant of $f(z)$ is a perfect square in $\mathbb{Q}$.

THEOREM 2.4. If $q_{3}(z)$ is irreducible over $\mathbb{Q}$, then its Galois group $\mathbb{G}\left(q_{3}\right)$ is isomorphic neither to the cyclic group $C_{7}$ nor to the metacyclic group $M_{7}$. Thus, $q_{3}(z)$ is not solvable by radicals.

Proof. Observing that the discriminant

$$
\Delta=2(y+4 x)^{4} y^{4} a^{6} x^{4}\left(y+a^{2}+x\right)^{6} \bmod 5
$$

of $q_{3}(z)$ is not a quadratic residue modulo 5 , because 2 is not, then $\Delta$ cannot be a perfect square in $\mathbb{Q}$. It follows by Proposition 2.3 that $\mathbb{G}\left(q_{3}\right)$ is not a subgroup of $A_{7}$.

Recalling that an irreducible polynomial of prime degree is solvable by radicals if and only if its Galois group is either a cyclic or a metacyclic group [1], the theorem is proved by a contradiction: both $C_{7}$ and $M_{7}$ are subgroups of the alternating group $A_{7}$ since 7 is an odd prime and cyclic permutations of odd length are even, that is, they belong to the alternating group.

2.1. Optimal simplexes in dimension $n>3$. The result that $p_{n}(t)$ is unsolvable by radicals can be proved for every $n$, considering special points $A \in \mathbb{R}^{n}$. For instance, assuming $a_{1}=1, a_{2}=4$, and $a_{3}=a_{4}=\cdots=a_{n}=6$, the positive 
TABLE 2.1. Primes $p_{2}$ and $p_{6}$ identifying 2- and 6-cycles in $\mathfrak{G}\left(p_{n}(t)\right)$.

\begin{tabular}{ccr}
\hline$n$ & $p_{6}$ & $p_{2}$ \\
\hline 4 & 41 & 3803 \\
5 & 17 & 5 \\
6 & 19 & 1439 \\
7 & 37 & 2281 \\
8 & 29 & 2971 \\
9 & 11 & 6619 \\
10 & 73 & 7 \\
11 & 11 & 31 \\
12 & 13 & 61 \\
13 & 41 & 1259 \\
14 & 41 & 3121 \\
15 & 23 & 1459 \\
\hline
\end{tabular}

root of $p_{n}(t)$ is the root of a factor polynomial $f_{7}(t)$ of degree 7 :

$$
\begin{aligned}
f_{7}(t)= & (n-1)^{8} t^{7}+848(n-2)(n-1)^{6} t^{6} \\
& +32\left(612 n^{3}+3547 n^{2}-20888 n+22644\right)(n-1)^{4} t^{5} \\
& +256\left(33012 n^{4}-177667 n^{3}+299918 n^{2}-126444 n-53776\right)(n-1)^{2} t^{4} \\
& +2^{8}\left(32000896 n+395280 n^{6}+9437312 n^{3}+3118673 n^{4}\right. \\
& \left.\quad-31326888 n^{2}-2446776 n^{5}-11090288\right) t^{3} \\
& +2^{15}\left(-7415124+19258460 n+8716105 n^{3}\right. \\
& \left.\quad-1777968 n^{4}-18997599 n^{2}+112752 n^{5}\right) t^{2} \\
& +2^{18}\left(-27802116+67636164 n+26141625 n^{3}\right. \\
& \left.\quad-4641624 n^{4}-62170322 n^{2}+198288 n^{5}\right) t \\
& -641728512(36 n-55)(n-2)(9 n-14)(36 n-71) .
\end{aligned}
$$

Applying the van der Waerden technique, with the primes given in Table 2.1, the Galois group of $f_{7}(t)$ is $S_{7}$ for $4 \leq n \leq 15$.

Unfortunately, it remains unproven that the same technique works for every $n$ without exception.

3. Solutions by radicals. As a consequence of Theorems 2.1 and 2.4, polynomial $q_{3}(z)$ is solvable by radicals only when it splits over the coefficient field. This factoring certainly occurs if $c=b$, in which case we have

$$
q_{3}(z)=\left(z^{3}-2 a z^{2}+a^{2} z+4 a x\right)\left(2 x-a z+z^{2}\right)^{2},
$$


so that solving the cubic equation $z^{3}-2 a z^{2}+a^{2} z+4 a x=0$ for $z$, and computing $t$, we obtain

$$
\begin{aligned}
& t=\frac{a^{2}}{3}\left(\sqrt[3]{1+36 \frac{b^{2}}{a^{2}}+216 \frac{b^{4}}{a^{4}}+24 \frac{b^{3}}{a^{3}} \sqrt{3+81 \frac{b^{2}}{a^{2}}}}\right.-2) . \\
&\left.+\frac{1+24\left(b^{2} / a^{2}\right)}{\sqrt[3]{1+36\left(b^{2} / a^{2}\right)+216\left(b^{4} / a^{4}\right)+24\left(b^{3} / a^{3}\right) \sqrt{3+81\left(b^{2} / a^{2}\right)}}}\right) .
\end{aligned}
$$

From now on, $a, b$, and $c$ are assumed to be distinct rational numbers. The most meaningful situation concerns the splitting of $q_{3}(z)$ with at least one linear factor. To analyze this case, it is convenient to assume $a$ and $z$ to be parameters and to consider $q_{3}(z)=Q(x, y, a, z)=0$ as a defining equation of a cubic curve $\mathscr{C}_{3}$ with respect to variables $x$ and $y$.

A straightforward calculation shows that $\mathscr{C}_{3}$ is a singular cubic with a double point

$$
P_{D}=\left(\frac{a z-z^{2}}{2}, \frac{a z-z^{2}}{2}\right)
$$

Therefore, a rational representation for $\mathscr{C}_{3}$ is obtained considering the intersection with a straight line through $P_{D}$ and slope $n / m$,

$$
x=\frac{a z-z^{2}}{2}+m u, \quad y=\frac{a z-z^{2}}{2}+n u .
$$

The value of $u \neq 0$ identifies the third intersection of the line with the cubic

$$
\begin{aligned}
u= & -\frac{1}{8 a m n(m+n)} \\
& \times z\left(z^{2} m^{2}+z^{2} n^{2}+4 m^{2} a^{2}+4 n^{2} a^{2}-4 a z n^{2}\right. \\
& \left.\quad-8 a z m n+2 z^{2} m n-4 a z m^{2}+4 m a^{2} n\right)
\end{aligned}
$$

so that points on $\mathscr{C}_{3}$ have the parametric representation

$$
x=\frac{-z(2 m a-n z-z m)^{2}}{8 a n(n+m)}, \quad y=\frac{-z(2 n a-n z-z m)^{2}}{8 a m(n+m)} .
$$

Setting $z=-2 a \kappa$, we have $t=4 a^{2}\left(\kappa^{2}+\kappa\right)$ and, correspondingly, polynomial $q_{3}(z)$ has a linear factor $z+2 a \kappa$. Recalling that $b=\sqrt{x}$ and $c=\sqrt{y}$, we have

$$
A=\left(a, a(m+n \kappa+\kappa m) \sqrt{\frac{\kappa}{n(n+m)}}, a(n+n \kappa+\kappa m) \sqrt{\frac{\kappa}{m(n+m)}}\right)
$$


as a function of $\kappa, m, n$, and $a$. The vertex coordinates of the optimal triangle are

$$
\begin{aligned}
& v_{1}=2 a(\kappa+1), \\
& v_{2}=2 a(\kappa+1)(m+n) \sqrt{\frac{\kappa}{n(n+m)},} \\
& v_{3}=2 a(\kappa+1)(m+n) \sqrt{\frac{\kappa}{m(n+m)}},
\end{aligned}
$$

and the area is

$$
S=2 a^{2}(\kappa+1)^{2}(m+n) \sqrt{\frac{\kappa(\kappa+1)}{m n}} .
$$

4. Optimal Diophantine triangles. Minimal triangles having rational vertex coordinates $v_{1}, v_{2}$, and $v_{3}$, and possibly rational areas $S$, are called optimal Diophantine triangles. It is evident that points $A$, with every coordinate being a nonzero rational number, and optimal Diophantine triangles are obtained by placing rational parameter values into (3.7) and (3.8), respectively. Moreover, the next theorem shows that every optimal Diophantine triangle with nonzero vertices is obtained in this way.

THEOREM 4.1. Let A be a point of the first orthant with rational coordinates, none of which equals 0 . Every optimal Diophantine triangle with vertices $V_{1}, V_{2}$, and $V_{3}$ is originated by a point with coordinates

$$
A=\left(\vartheta \mu_{1} \mu_{2}\left(\lambda_{1}^{2} \mu_{2}^{2}+\lambda_{2}^{2} \mu_{1}^{2}\right), \vartheta \mu_{2} \lambda_{1}^{3}\left(\lambda_{2}^{2}+\mu_{2}^{2}\right), \vartheta \mu_{1} \lambda_{2}^{3}\left(\lambda_{1}^{2}+\mu_{1}^{2}\right)\right),
$$

where $\vartheta$ is a rational number and $\lambda_{1}, \lambda_{2}, \mu_{1}$, and $\mu_{2}$ are integers. The vertex coordinates are

$$
\begin{aligned}
& v_{1}=2 \vartheta \mu_{1} \mu_{2}\left(\lambda_{1}^{2} \lambda_{2}^{2}+\lambda_{1}^{2} \mu_{2}^{2}+\lambda_{2}^{2} \mu_{1}^{2}\right), \\
& v_{2}=2 \vartheta \lambda_{1} \mu_{2}\left(\lambda_{1}^{2} \lambda_{2}^{2}+\lambda_{1}^{2} \mu_{2}^{2}+\lambda_{2}^{2} \mu_{1}^{2}\right), \\
& v_{3}=2 \vartheta \lambda_{2} \mu_{1}\left(\lambda_{1}^{2} \lambda_{2}^{2}+\lambda_{1}^{2} \mu_{2}^{2}+\lambda_{2}^{2} \mu_{1}^{2}\right) .
\end{aligned}
$$

Correspondingly, the positive root $t$ and area $S$ are

$$
\begin{aligned}
& t=4 \vartheta^{2} \lambda_{1}^{2} \lambda_{2}^{2} \mu_{1}^{2} \mu_{2}^{2}\left(\lambda_{1}^{2} \lambda_{2}^{2}+\lambda_{1}^{2} \mu_{2}^{2}+\lambda_{2}^{2} \mu_{1}^{2}\right), \\
& S=2 \vartheta^{2} \mu_{1} \mu_{2}\left(\lambda_{1}^{2} \lambda_{2}^{2}+\lambda_{1}^{2} \mu_{2}^{2}+\lambda_{2}^{2} \mu_{1}^{2}\right)^{5 / 2} .
\end{aligned}
$$

Proof. Since $v_{1}$ is rational by assumption, then $z=v_{1}$ is a rational root of $q_{3}(z)$. It follows that every rational triple $\left\{v_{1}, v_{2}, v_{3}\right\}$ admits the parametric representation (3.8). Furthermore, rational $v_{1}$ and $a$ imply rational $\kappa$, while rational $v_{2}$ and $v_{3}$ require that

$$
\kappa\left(1+\frac{m}{n}\right)=\frac{\lambda_{1}^{2}}{\mu_{1}^{2}}, \quad \kappa\left(1+\frac{n}{m}\right)=\frac{\lambda_{2}^{2}}{\mu_{2}^{2}},
$$


where $\lambda_{1}, \lambda_{2}, \mu_{1}$, and $\mu_{2}$ are integers. It follows that

$$
\frac{m}{n}=\left(\frac{\mu_{2} \lambda_{1}}{\mu_{1} \lambda_{2}}\right)^{2}, \quad \kappa=\frac{\lambda_{1}^{2} \lambda_{2}^{2}}{\lambda_{1}^{2} \mu_{2}^{2}+\lambda_{2}^{2} \mu_{1}^{2}} .
$$

In summary, setting $a=\vartheta \mu_{1} \mu_{2}\left(\lambda_{1}^{2} \mu_{2}^{2}+\lambda_{2}^{2} \mu_{1}^{2}\right)$ in

$$
A=\left(a, a \frac{\lambda_{1}^{3}\left(\lambda_{2}^{2}+\mu_{2}^{2}\right)}{\mu_{1}\left(\lambda_{1}^{2} \mu_{2}^{2}+\lambda_{2}^{2} \mu_{1}^{2}\right)}, a \frac{\lambda_{2}^{3}\left(\lambda_{1}^{2}+\mu_{1}^{2}\right)}{\mu_{2}\left(\lambda_{1}^{2} \mu_{2}^{2}+\lambda_{2}^{2} \mu_{1}^{2}\right)}\right),
$$

we obtain the representation (4.1) for $A$, where possible integer denominators are included in rational $\vartheta$. In conclusion, $S$ and (4.2) are obtained directly from (3.9) and (3.8), respectively.

4.1. Integer solutions. Let $\mathscr{T}(A)$ be an optimal Diophantine triangle having integer vertex coordinates with the restriction that $A(a, b, c)$ is a point having integer coordinates. Any triangle obtained by scaling the coordinates of $\mathscr{T}(A)$ with an integer factor $\lambda$ is minimal for a point $B(\lambda a, \lambda b, \lambda c)$ obtained by scaling the $A$ coordinates of the same factor. Therefore, solutions with relatively prime point coordinates are of most interest. Any point $A$ with $a, b$, and $c$ relatively prime integers is said to be a primitive point and, correspondingly, $\mathscr{T}(A)$ is called a primitive optimal Diophantine triangle. Every primitive optimal Diophantine triangle is given by the following theorem, whose proof stems from Theorem 4.1 .

THEOREM 4.2. The integer coordinates of every primitive point $A$, associated to an optimal Diophantine triangle, are given by (4.1), with a possible coordinate permutation, where the parameters $\lambda_{1}, \mu_{1}, \lambda_{2}$, and $\mu_{2}$ are integers satisfying the conditions $\operatorname{gcd}\left(\lambda_{1}, \mu_{1}\right)=\operatorname{gcd}\left(\lambda_{2}, \mu_{2}\right)=\operatorname{gcd}\left(\lambda_{1}, \lambda_{2}\right)=\operatorname{gcd}\left(\mu_{1}, \mu_{2}\right)=1$, and either $\vartheta=1$, if at least one of the four parameters is even in which case $v_{1}, v_{2}$, and $v_{3}$ are even integers, or $\vartheta=1 / 2$, if all four parameters are odd.

PROoF. The coordinates of $A$ are relatively prime if $\vartheta$ is chosen to cancel the greatest common factor

$$
G=\operatorname{gcd}\left\{\mu_{1} \mu_{2}\left(\lambda_{1}^{2} \mu_{2}^{2}+\lambda_{2}^{2} \mu_{1}^{2}\right), \mu_{2} \lambda_{1}^{3}\left(\lambda_{2}^{2}+\mu_{2}^{2}\right), \mu_{1} \lambda_{2}^{3}\left(\lambda_{1}^{2}+\mu_{1}^{2}\right)\right\}
$$

Let the conditions $\operatorname{gcd}\left(\lambda_{1}, \mu_{1}\right)=\operatorname{gcd}\left(\lambda_{2}, \mu_{2}\right)=1$ be assumed to avoid trivial common factors. Furthermore, assuming $\operatorname{gcd}\left(\mu_{1}, \mu_{2}\right)=1$ and $\operatorname{gcd}\left(\lambda_{1}, \lambda_{2}\right)=1$, the factors $\mu_{1}, \mu_{2}, \lambda_{1}^{3}$, and $\lambda_{2}^{3}$ are dropped, computing $G$. Thus, subtracting the second term multiplied by $\lambda_{1}^{2}$ from the first term in

$$
G=\operatorname{gcd}\left\{\left(\lambda_{1}^{2} \mu_{2}^{2}+\lambda_{2}^{2} \mu_{1}^{2}\right),\left(\lambda_{2}^{2}+\mu_{2}^{2}\right),\left(\lambda_{1}^{2}+\mu_{1}^{2}\right)\right\},
$$


we obtain

$$
\begin{aligned}
G & =\operatorname{gcd}\left\{\left(\lambda_{2}^{2} \mu_{1}^{2}-\lambda_{1}^{2} \lambda_{2}^{2}\right),\left(\lambda_{2}^{2}+\mu_{2}^{2}\right),\left(\lambda_{1}^{2}+\mu_{1}^{2}\right)\right\} \\
& =\operatorname{gcd}\left\{\left(\mu_{1}^{2}-\lambda_{1}^{2}\right),\left(\lambda_{2}^{2}+\mu_{2}^{2}\right),\left(\lambda_{1}^{2}+\mu_{1}^{2}\right)\right\}
\end{aligned}
$$

where the $\lambda_{2}^{2}$ factor in the first term which is relatively prime with $\left(\lambda_{2}^{2}+\mu_{2}^{2}\right)$ has been dropped. Adding the last term to the first one, we get $G=\operatorname{gcd}\left\{2 \mu_{1}^{2},\left(\lambda_{2}^{2}+\right.\right.$ $\left.\left.\mu_{2}^{2}\right),\left(\lambda_{1}^{2}+\mu_{1}^{2}\right)\right\}$, which shows that $G$ is either 1 or 2 if and only if the four parameters are odd.

Since (4.1) was obtained referring to the first coordinate $a$ of point $A$, when we are looking for every integer solution, it is necessary to refer to the similar solutions obtained considering the $b$ and then the $c$ coordinate. This is tantamount to a permutation of the coordinates of any integer solution obtained with $a$.

Note that it is possible to have $\mathscr{T}(A)$ with integer vertex coordinates and $A$ having rational coordinates. For example, taking $A(75 / 13,17 / 13,45 / 26)$, the corresponding optimal triangle has integral vertices $v_{1}=12, v_{2}=4$, and $v_{3}=3$.

Although $a=0$ is not included in Theorem 4.2, in this case any point $A$ with $b$ and $c$ integers has an optimal triangle with integer vertex coordinates $v_{1}=0$, $v_{2}=2 b$, and $v_{3}=2 c$ and area $S=2 b c$.

The integer solutions with $c=b$ are obtained directly from (4.1) and (4.2), with $\lambda_{1} / \mu_{1}=\lambda_{2} / \mu_{2}=\lambda / \mu, \kappa=\left(\lambda^{2} / 2 \mu^{2}\right)$, and $a=29 \mu^{3}$. We have

$$
\begin{gathered}
A=\left(2 \vartheta \mu^{3}, \vartheta \lambda\left(\lambda^{2}+\mu^{2}\right), \vartheta \lambda\left(\lambda^{2}+\mu^{2}\right)\right), \quad t=4 \vartheta^{2} \lambda^{2} \mu^{2}\left(\lambda^{2}+2 \mu^{2}\right), \\
v_{1}=2 \vartheta \mu\left(\lambda^{2}+2 \mu^{2}\right), \quad v_{2}=v_{3}=2 \vartheta \lambda\left(\lambda^{2}+2 \mu^{2}\right)
\end{gathered}
$$

and the area is

$$
S=29^{2} \lambda\left(\sqrt{\lambda^{2}+2 \mu^{2}}\right)^{5} .
$$

Solutions with integer $S$ are obtained when $\lambda^{2}+2 \mu^{2}=w^{2}$ is a square of an integer $w$. A general solution of this quadratic equation is

$$
\mu=\varrho 2 u v, \quad \lambda=\varrho\left(2 u^{2}-v^{2}\right), \quad w=\varrho\left(2 u^{2}+v^{2}\right),
$$

with $u$ and $v$ relatively prime [2]. 
Every primitive integer solution is given by Theorem 4.2; particular choices of these parameters yield interesting integer solutions. For example, with $\mu_{1}=$ $\lambda_{2}=1, \lambda_{2}=\alpha$, and $\mu_{2}=\mu$, we have

$$
\begin{aligned}
A & =\left(\mu\left(1+\mu^{2} \alpha^{2}\right), \mu \alpha^{3}\left(1+\mu^{2}\right),\left(1+\alpha^{2}\right)\right), \\
t & =4 \mu^{2} \alpha^{2}\left(1+\alpha^{2}+\mu^{2} \alpha^{2}\right),
\end{aligned}
$$

and $v_{1}=2 \mu\left(1+\alpha^{2}+\mu^{2} \alpha^{2}\right), v_{2}=2 \mu \alpha\left(1+\alpha^{2}+\mu^{2} \alpha^{2}\right)$, and $v_{3}=2\left(1+\alpha^{2}+\mu^{2} \alpha^{2}\right)$.

Furthermore, the area of the triangle $S=2 \mu \sqrt{\left(1+\alpha^{2}+\mu^{2} \alpha^{2}\right)^{5}}$ is an integer if $1+\alpha^{2}+\mu^{2} \alpha^{2}=\gamma^{2}$, equivalently $\gamma^{2}-\left(1+\mu^{2}\right) \alpha^{2}=1$. One solution is $\alpha=2 \mu$ and $\gamma=2 \mu^{2}+1$, and for any $\mu$, we have an infinity of solutions from the expression

$$
\gamma_{n}+\alpha_{n} \sqrt{1+\mu^{2}}=\left(\left(2 \mu^{2}+1\right)+2 \mu \sqrt{1+\mu^{2}}\right)^{n} .
$$

For example, the smallest solution having distinct $a, b$, and $c$ is obtained with $\mu=2$. We have $\alpha_{n}=F_{6 n} / 2$, where $F_{n}$ denotes a Fibonacci number, and

$$
A=\left(1+\left(\frac{F_{6 n}}{2}\right)^{2}, 10\left(\frac{F_{6 n}}{2}\right)^{3}, 2\left(1+4\left(\frac{F_{6 n}}{2}\right)^{2}\right)\right)
$$

Some initial integer values of $\alpha$ are 4, 72, and 1292, to which correspond points $A$

$(17,640,130),(5185,3732480,41474),(1669265,21566890880,13354114)$

and areas 236196, 432702467204, 805003373860133796, respectively.

5. Conclusions. The problem of finding a simplex with minimum volume and passing through a given point belongs to that sort of the elementary problem made famous by the three classical Greek problems and it is solved using the same methods.

Specifically, in this paper, it has been proved that the solution of the optimal simplex problem depends on the positive root of a $\left(2^{n}-1\right)$-degree polynomial. This polynomial cannot be solved using radicals for any $n$ from 3 up to 15 when the coordinates of $A$ are transcendental over $\mathbb{Q}$. It is likely that it cannot be solved by radicals for any $n$, although a proof has not been found. Limited to dimension $n=3$ and points $A$ of distinct integer coordinates, it has been shown that if the polynomial $p_{3}(t)$ of degree 7 is irreducible, then it is unsolvable by radicals. A parametric representation of every integer solution, which corresponds to polynomials $p_{3}(t)$ that split with a linear factor, has been obtained. 
ACKNOWLEDGMENTS. I would like to thank the referee for some very constructive criticism that improved the formulation of the paper. A preliminary version of this paper was presented at the Tenth International Conference on Fibonacci Numbers and Their Applications, 24-28 June 2002, Flagstaff, Arizona, USA.

\section{REFERENCES}

[1] R. Bourgne and J.-P. Azra, Écrits et Mémoires Mathématiques d'Évariste Galois, Édition Critique Intégrale de ses Manuscrits et Publications. Préface de J. Dieudonné, Gauthier-Villars \& Cie, Imprimeur-Éditeur-Libraire, Paris, 1962.

[2] L. E. Dickson, Introduction to the Theory of Numbers, Dover Publications, New York, 1957.

[3] J.-P. Escofier, Galois Theory, Graduate Texts in Mathematics, vol. 204, SpringerVerlag, New York, 2001.

[4] C. F. Gauss, Disquisitiones Arithmeticae, Springer-Verlag, New York, 1986.

[5] C. A. Neff, Finding the distance between two circles in three-dimensional space, IBM J. Res. Develop. 34 (1990), no. 5, 770-775.

[6] M. Radić and T. K. Pogány, Algebraic equations connected with tangential polygons and their solvability by radicals, Appl. Math. E-Notes 1 (2001), 118123.

[7] D. M. Y. Sommerville, An Introduction to the Geometry of $n$ Dimensions, Dover Publications, New York, 1958.

[8] D. Spring, Solution of a calculus problem on minimal volume, Amer. Math. Monthly 108 (2001), no. 3, 217-221.

[9] I. Stewart, Galois Theory, Chapman \& Hall Mathematics, New York, 1998.

[10] B. L. van der Waerden, Modern Algebra, Vol. I and II, Frederick Ungar Publishing, New York, 1966.

Michele Elia: Dipartimento di Elettronica, Politecnico di Torino, Corso Duca degli Abruzzi 24, 10129 Torino, Italy

E-mail address: e1 ia@po1ito.it 


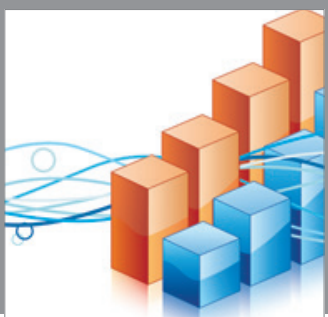

Advances in

Operations Research

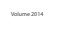

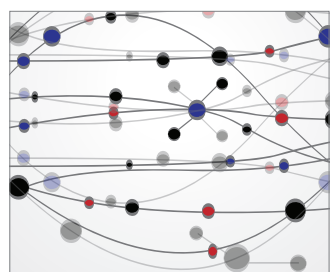

\section{The Scientific} World Journal
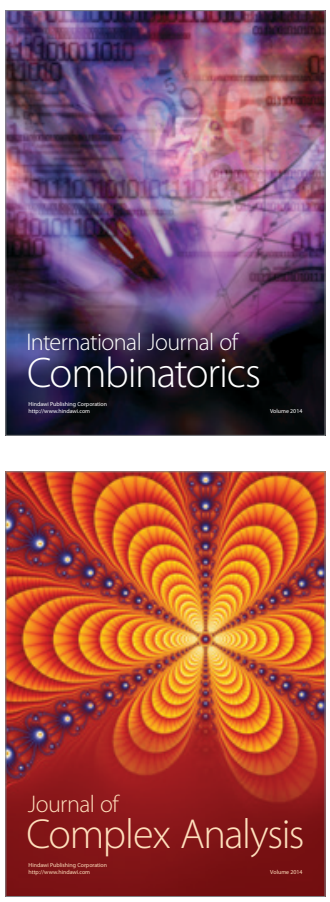

International Journal of

Mathematics and

Mathematical

Sciences
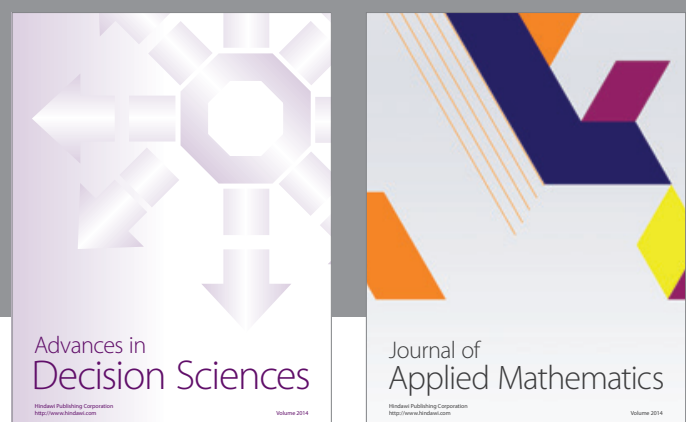

Journal of

Applied Mathematics
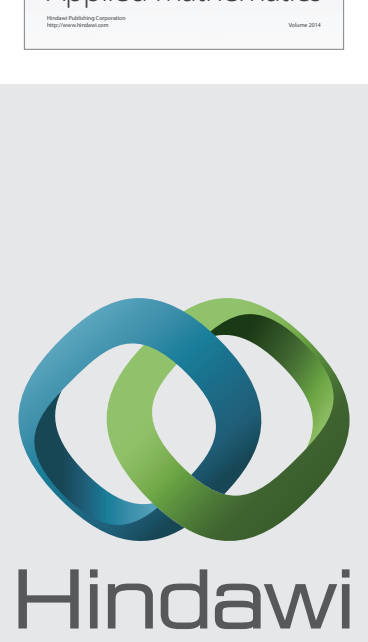

Submit your manuscripts at http://www.hindawi.com
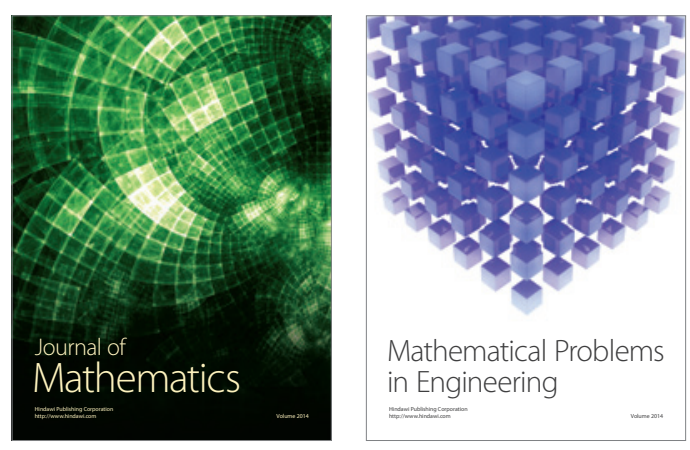

Mathematical Problems in Engineering
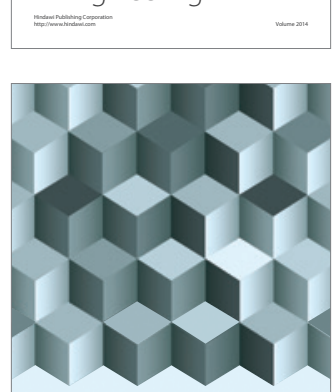

Journal of

Function Spaces
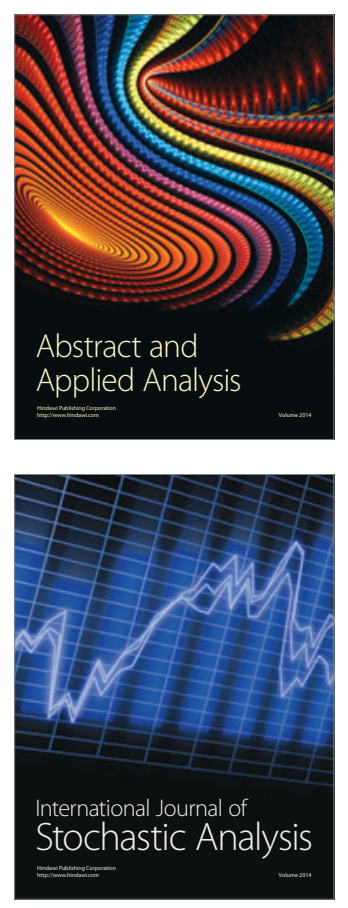

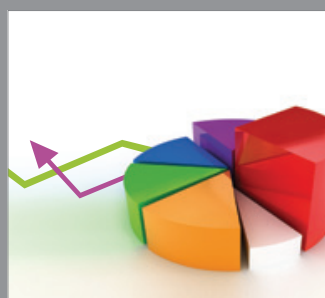

ournal of

Probability and Statistics

Promensencen
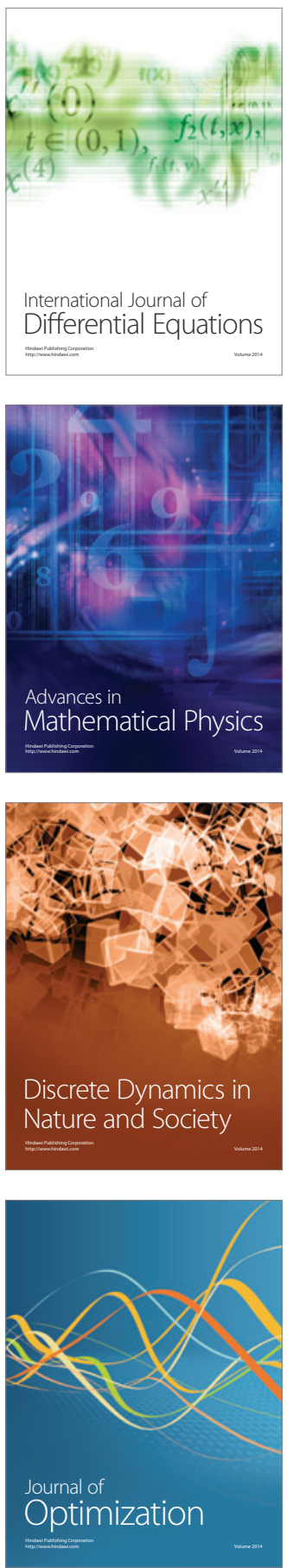